Winter Meeting, 2 December 2010, Functional genomic biomarkers, nutrition and disease susceptibility

\title{
Transcriptomic analysis of host intestinal tissue to assess the consequences of dietary protein and secondary infection on immunity to nematodes
}

\author{
S. Athanasiadou ${ }^{1}$, L. A. Jones ${ }^{1}$, S. T. G. Burgess ${ }^{2}$, A. D. Pemberton ${ }^{3}$, I. Kyriazakis ${ }^{1,4}$, J. F. Huntley ${ }^{2}$ \\ and J. G. M. Houdijk ${ }^{1}$ \\ ${ }^{1}$ Scottish Agricultural College, EH9 3JG, UK, ${ }^{2}$ Moredun Research Institute, EH26 OPZ, UK, ${ }^{3}$ University of Edinburgh, \\ EH25 9RG, UK and ${ }^{4}$ University of Thessaly, 43100, Greece
}

Periparturient breakdown of immunity to nematodes renders animals susceptible to infection and a source of infection for their offspring. Protein supplementation can reduce associated elevated parasite burdens ${ }^{(1)}$; however, the underlying mechanism is unclear. Here, we used a genome-wide approach to assess the effects of protein supplementation and secondary infection, on gene expression in the small intestine of lactating rats during nematode expulsion.

Previously immune, lactating rats $(n$ 6) were either sham infected or re-infected with 1600 Nippostrongylus brasiliensis infective larvae, and were restrictedly fed iso-energetic foods with low $(130 \mathrm{~g} \mathrm{CP} / \mathrm{kg} \mathrm{DM})$ or high $(270 \mathrm{~g} \mathrm{CP} / \mathrm{kg} \mathrm{DM})$ protein content. At post-mortem intestinal tissue was fixed for RNA extraction. The Affymetrix Rat (Gene 1.0 ST) array was used for gene expression analysis. Data normalisation, clustering and statistical analysis was performed in Genespring GX 11.0; function, network and pathway analysis was performed with Ingenuity Pathway Analysis. Statistical significance was determined by a two-way ANOVA, with dietary protein and secondary infection as factors.

Secondary infection significantly regulated the expression of 91 genes; top affected functions included immune cell trafficking and cell-mediated immune responses. Genes with a previously described role in immunity to nematodes in various infection models were identified. Other genes, such as $A G R 2$ and $G 6 P C$, were for the first time associated with worm expulsion.

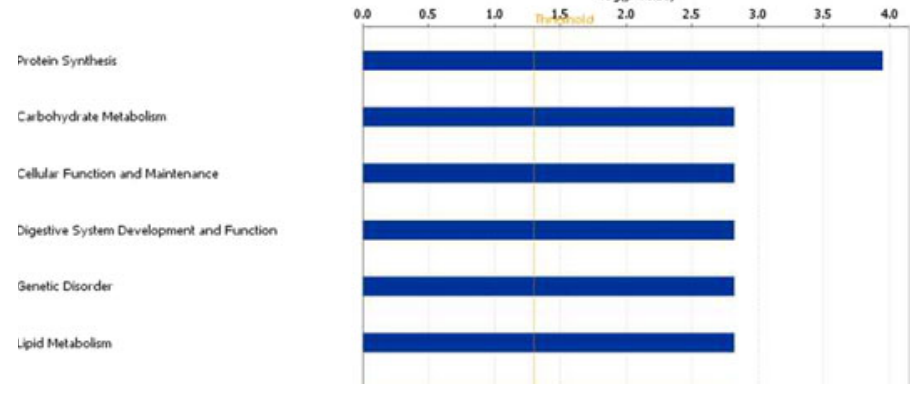

Fig. 1. Top biological functions affected by protein supplementation in lactating rats.

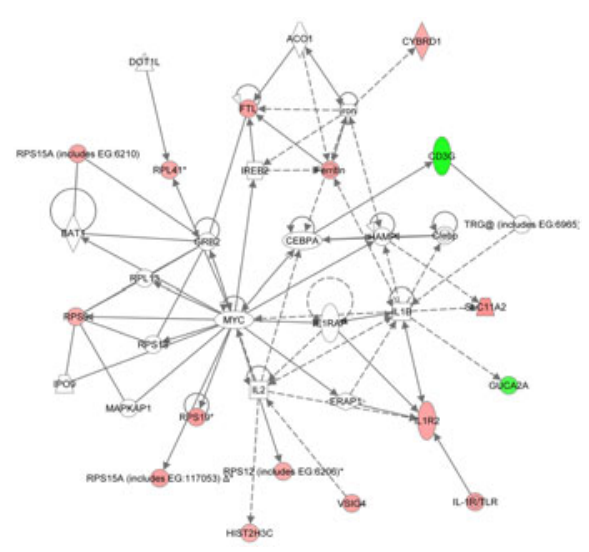

Fig. 2. Gene network affected by protein supplementation in lactating rats (red - up- and green - down-regulated expressions).

Protein supplementation, which reduced worm burdens by $70 \%^{(2)}$, significantly regulated expression of 64 genes; for top affected functions see Fig. 1 . It increased cell metabolism and turnover, evident from the high number of non-coding RNA transcripts and the increased synthesis of ribosomal proteins (Fig. 2). It also induced responses related to adaptive immune responses, through T-cell activation and proliferation (ILIR2 and VSIG4, respectively). The up-regulation of transcription regulator FOXP1 in un-supplemented infected rats may indicate delayed immune response. The study provides the first evidence for nutritional regulation of genes related to immunity to nematodes at transcript level; their role is currently investigated.

1. Houdijk JG, Jessop NS, Knox DP et al. (2005) Br J Nutr 93, 493-499.

2. Sakkas P, Jones L, Houdijk JG et al. (2009) Proc Nutr Soc 68, E136. 